\title{
Effects of the canine rattlesnake vaccine in moderate to severe cases of canine crotalid envenomation
}

This article was published in the following Dove Press journal:

Veterinary Medicine: Research and Reports

31 October 2014

Number of times this article has been viewed

\author{
McGee J Leonard' \\ Catherine Bresee ${ }^{2}$ \\ Andrew Cruikshank' \\ 'Animal Specialty and Emergency \\ Center, Los Angeles, CA, USA; ${ }^{2}$ The \\ Biostatistics and Bioinformatics \\ Research Center, Cedars-Sinai Medical \\ Center, Los Angeles, CA, USA
}

\begin{abstract}
This is a retrospective multicenter study (2006-2012) examining a population of dogs with moderate to severe crotalid envenomation for protective effects of the canine rattlesnake vaccine. Five nonacademic emergency and referral veterinary hospitals in Southern California were involved in the study and contributed records regarding a total of 82 client-owned dogs that were treated for naturally occurring rattlesnake envenomation. All dogs received antivenin (Crotalidae) polyvalent, with dosages ranging from one to three vials (mean: $1.3 \pm 0.6$ ). Fourteen dogs $(17 \%)$ had a history of prior vaccination against crotalid venom. In univariate logistic regression modeling, cases with lower body weight $(P=0.0001)$ or higher snakebite severity scores $(P<0.0001)$ were associated with greater morbidity. No statistically significant difference in morbidity or mortality between vaccinated and unvaccinated dogs was found. The findings of this study did not identify a significantly protective effect of previous vaccination in the cases of moderate to severe rattlesnake envenomation that require treatment with antivenin.
\end{abstract}

Keywords: rattlesnake envenomation, vaccine, antivenin, canine

\section{Introduction}

Rattlesnake envenomation is a common presenting complaint at veterinary clinics in rattlesnake-endemic areas. Approximately 120 species of snakes are found in the United States, with 20 of these considered venomous. ${ }^{1}$ Venomous snakes are commonly encountered in the southern United States, with bites occurring most frequently in warmer months when snakes are most active. Coral snakes, of the family Elapidae, are found in the southeastern United States and are responsible for only a small percentage of envenomations, largely due to their nonaggressive and reclusive nature. ${ }^{2}$ Pit vipers, members of the Crotalinae subfamily of snakes, are most frequently associated with envenomation of canines. Genera of pit vipers found in the United States include Crotalus, Agkistrodon, and Sistrurus. ${ }^{3}$

The most prevalent rattlesnake endemic to the geographic region of this study is the western diamondback rattlesnake, Crotalus atrox. Its venom is commonly associated with development of coagulopathies. Although the specific composition of venom varies between individual snakes and between geographic locations, C. atrox venom is characterized by the disintegrin crotoxin. ${ }^{4} \mathrm{High}$ metalloproteinase activity is also common. Similar to other crotalid venoms, western diamondback rattlesnake's venom contains isoenzymes of phospholipase A, which have been associated with release of histamine, kinins, serotonin, and acetylcholine, and have been implicated in neurotoxicity. ${ }^{5}$
Correspondence: McGee J Leonard 1535 South Sepulveda Boulevard, Los Angeles, CA, USA

Tel $+\mid 310473$ I56 |

Fax + I 3104798976

Email mleonard@asecvets.com
Veterinary Medicine: Research and Reports 20I4:5 I53-I58 153

Dovepress

http://dx.doi.org/10.2147/VMRR.S69216 (c) (i) (-) 2014 Leonard et al. This work is published by Dove Medical Press Limited, and licensed under Creative Commons Attribution - Non Commercial (unported, v3.0) License. The full terms of the License are available at http://creativecommons.org/licenses/by-nc/3.0/. Non-commercial uses of the work are permitted without any further permisson how to request permission may be found at: http://www.dovepress.com/permissions.php 
Antivenin is the only specific therapy available for rattlesnake envenomation. Its administration is common in human medicine and, in many regions, has become the standard of care for moderate to severe cases of canine envenomation. Antivenin products are composed of whole or partial immunoglobulin proteins that are produced in response to various components of rattlesnake venom. The goal of antivenin treatment is to infuse a complement of antibodies into an envenomated patient to neutralize circulating venom components. ${ }^{6}$ First generation antivenin products were made from whole IgG molecules. The Fc fragment included in the immunoglobulin molecule is involved in complement binding and subsequent cytotoxicity and thus may be implicated in adverse immune responses including immediate hypersensitivity reactions. ${ }^{7}$ Since the Fc fragments are not necessary for antigen binding, newer antivenin products have been developed consisting of virtually pure IgG Fab fragments. ${ }^{8}$

Antivenin (Crotalidae) polyvalent (ACP) (Boehringer Ingelheim Vetmedica, Inc., St Joseph, MO, USA) is produced by inoculating horses with venom of the eastern and western diamondback rattlesnakes, the tropical rattlesnake, and the Fer-de-lance. ${ }^{8}$ This antivenin consists of whole IgG molecules. ACP is no longer produced for human use in the United States, but it remains common in the veterinary field. Specific dosage recommendations for ACP are lacking. Envenomated humans routinely receive multiple doses of antivenin early in the treatment. ${ }^{1}$ The use of multiple vials of antivenin may quickly become cost prohibitive in veterinary patients. ACP manufacturer's recommendations are from one to five vials per dog, depending on the dog and the clinical presentation. The manufacturer also states that higher doses may be needed for smaller dogs due to their greater relative body surface area. ${ }^{9}$ One vial of ACP is often sufficient to slow or halt progression of clinical signs. General guidelines for use of antivenin products recommend treatment within 4 hours of envenomation, although some positive effect may be expected after 24 hours or more. ${ }^{2}$

A western diamondback (C. atrox) toxoid vaccine was licensed for use in the state of California in 2003 and released nationally in 2004. A canine and an equine version of the crotalid toxoid vaccine are currently produced by a single manufacturer (Red Rock Biologics, Woodland, CA, USA). The goal of vaccination is to stimulate the patient to form a full complement of active antibodies against various components of the venom, which may then be mobilized acutely in the case of envenomation. ${ }^{10}$ Manufacturer claims include crossprotection against prairie, Great Basin, Northern and Southern Pacific, and timber rattlesnakes, as well as the sidewinder, massasauga, and copperhead snakes. Some protection against the eastern diamondback rattlesnake venom may be produced but the vaccine manufacturer does not claim cross-protection against venom of cottonmouth, Mojave, or coral snakes. Manufacturer guidelines for vaccination recommend an initial vaccine followed by a booster 1 month later and annual boosters thereafter. Vaccines should be administered approximately 1 month prior to the start of the rattlesnake season, usually in mid to late winter. Additional boosters of the vaccine may be beneficial for dogs weighing less than 25 pounds due to their apparent increased sensitivity to rattlesnake venom, and also for dogs weighing more than 100 pounds, because they may mount a suboptimal antibody response to initial vaccination. ${ }^{10}$ Product information for the vaccine claims that all dogs bitten by rattlesnakes should be presented for emergent medical care, regardless of vaccination status, but that the vaccine may lessen or preclude the need for antivenin administration during treatment of envenomations. ${ }^{10}$

At the time of writing this manuscript, the only published studies investigating the efficacy of the vaccine were toxin neutralization studies conducted in mice at the time of vaccine development. In the most recent vaccine recommendations made by the American Animal Hospital Association, no position is taken regarding use of this vaccine, due to limited experimental and field data. ${ }^{11}$

The purpose of this study was to investigate the effects of vaccination on a population of moderately to severely envenomated dogs that required treatment with antivenin in Southern California and to determine whether a history of vaccination was associated with decreased morbidity or mortality.

\section{Materials and methods}

The medical records databases of five local emergency and referral veterinary hospitals were searched for dogs receiving antivenin therapy. Records were collected from July 2006 through March 2012; time periods for different hospitals varied based on the availability of searchable records. Cases were excluded if the final diagnosis was not consistent with rattlesnake envenomation or if complete records, including relevant laboratory data and case outcome, were not available. A total of 136 records were reviewed for study inclusion.

Medical records were reviewed by a single author (MJL) and the following data were collected: signalment, location of bite, vaccination status, body weight, vital signs at presentation (temperature, pulse rate, and respiration rate), physical examination findings, treatments instituted, number of vials of antivenin administered, length of hospitalization, and case 
outcome (discharge or death). Results of initial hematologic and coagulation evaluation including prothrombin time (PT), activated partial thromboplastin time (aPTT), and platelet count, as well as any follow-up testing performed during the course of treatment, were recorded.

A snakebite severity score (SSS) has been used in human medicine to quantify the severity of rattlesnake envenomation based on derangements of various body systems at presentation. ${ }^{12}$ This scoring system was later validated in cases of canine rattlesnake envenomation. ${ }^{13}$ Serial use of the SSS can aid in assessment of a patient's response to treatment following envenomation. ${ }^{13}$ An initial SSS was assigned to each patient in this study based on the collected data. The SSS was recalculated for each day of hospitalization and any progression of clinicopathologic abnormalities was recorded.

To attempt to quantify the morbidity or difficulty of case management for each patient through the duration of treatment, a morbidity scale was developed (Table 1). Each case was assigned a morbidity score (1-3) based on case progression and outcome. A case was classified as having low morbidity and received a score of 1 if a positive response to a single dose of antivenin was noted and the patient was discharged without complication in 24 hours or less. An additional point was added if, at any time during treatment, the SSS increased despite treatment with antivenin and/or if transfusion of any blood product was required. A maximal morbidity score of 3 was assigned when blood products were administered in addition to multiple doses of antivenin, or when a patient died during the course of treatment.

\section{Statistical methods}

All data were reported as means and standard deviations (SD) or counts and frequencies where appropriate. Continuous and normally distributed data were compared across groups by using Student's $t$-test or, failing normality, Wilcoxon rank-sum test. Categorical data were tested by using Fisher's exact test. Ordinal logistic regression methods were used

Table I Morbidity scale

\begin{tabular}{|c|c|}
\hline Morbidity score & Case features \\
\hline I (low) & $\begin{array}{l}\text { Routine presentation; adequate response to single } \\
\text { dose of ACP; no progression of SSS throughout } \\
\text { course of the treatment; discharge within } 24 \text { hours }\end{array}$ \\
\hline 2 (moderate) & $\begin{array}{l}\text { Increase of SSS after single ACP treatment or required } \\
\text { additional ACP dose/blood product transfusion }\end{array}$ \\
\hline 3 (high) & $\begin{array}{l}\text { Increase of SSS after single ACP treatment and } \\
\text { required additional ACP dose/blood product } \\
\text { transfusion; or patient expired despite treatment }\end{array}$ \\
\hline
\end{tabular}

Abbreviations: ACP, antivenin (crotalidae) polyvalent; SSS, snakebite severity score. to determine significant factors affecting morbidity scores. Linear regression methods were also performed to determine the number of days of hospital stay and number of antivenin vials. Multivariable modeling was conducted to adjust for a collective set of relevant clinical factors affecting morbidity scores, days of hospitalization, and number of antivenin vials. ${ }^{14}$ Values of $P<0.05$ were considered significant. All analysis was performed using SAS version 9.3.

\section{Results}

Of the 136 records reviewed, 82 dogs met the inclusion criteria for this study. Average age of dogs was $4.5 \pm 4.0$ years and their average weight was $24.9 \pm 11.9 \mathrm{~kg}$. Females were slightly overrepresented, with 47 females (57\%) and 35 males (43\%). Eleven percent of dogs were sexually intact (five males and four females). Most dogs (72\%) were bitten on the face or the muzzle. All dogs received one to three vials (mean 1.3 \pm 0.6 ) of antivenin. Fourteen dogs (17\%) had received the canine rattlesnake vaccine at some point prior to envenomation. A summary of case data for all dogs is given in Table 2 .

\section{Initial assessment}

All dogs in this study presented for evaluation within 6 hours of envenomation. Vital signs were recorded and a complete physical examination was performed for each dog at presentation. A complete blood count, PT, and aPTT were performed at the time of admission to the hospital.

Abnormalities in various body systems at presentation were recorded in order to assign an SSS to each dog. Eightytwo dogs $(100 \%)$ in this study had evidence of local tissue damage at the site of the bite, including punctures, ecchymoses, and swelling. Sixty-one dogs (74\%) had pulmonary abnormalities including tachypnea, dyspnea, and cyanosis. Forty dogs (49\%) had cardiovascular abnormalities including tachycardia, arrhythmias, and derangement of arterial blood pressure. Sixteen dogs (20\%) had abnormal findings related to the gastrointestinal system including abdominal pain, vomiting, and diarrhea. Thirty-eight dogs (46\%) had abnormal coagulation parameters including prolonged PT and aPTT times, thrombocytopenia, and clinically apparent abnormal hemorrhage or ecchymoses. Of the 82 dogs, only six dogs $(7 \%)$ demonstrated neurologic signs including weakness, ataxia, and lethargy. All the vaccinated dogs in this study presented with an SSS $\leq 5$.

\section{Treatment}

Every dog in the study was treated with ACP. Antivenin infusion monitoring was similar to a blood transfusion in all cases 
Table 2 Summary of case data

\begin{tabular}{|c|c|c|c|c|}
\hline & All cases $(\mathrm{N}=82)$ & Vaccinated $(n=14)$ & Unvaccinated $(n=68)$ & $P$-value \\
\hline Age & $4.5 \pm 4.0$ & $6.4 \pm 3.5$ & $5.2 \pm 4.1$ & $0.3163^{\#}$ \\
\hline Weight & $24.9 \pm 11.9$ & $29.9 \pm 12.4$ & $23.9 \pm 11.6$ & $0.0859^{\#}$ \\
\hline \multicolumn{5}{|l|}{ Sex, n (\%) } \\
\hline Male - intact & $5(6 \%)$ & $\mathrm{I}(7 \%)$ & $4(5 \%)$ & $0.8182 *$ \\
\hline Male - neutered & $30(37 \%)$ & $6(43 \%)$ & $24(35 \%)$ & \\
\hline Female - intact & $4(5 \%)$ & $0(0 \%)$ & $4(6 \%)$ & \\
\hline Female - spayed & $43(51 \%)$ & $6(43 \%)$ & $36(53 \%)$ & \\
\hline \multicolumn{5}{|l|}{ Bite location, n (\%) } \\
\hline Head & $59(72 \%)$ & $7(50 \%)$ & $52(76 \%)$ & $0.0563^{*}$ \\
\hline Body, limbs & $23(28 \%)$ & $7(50 \%)$ & $16(24 \%)$ & \\
\hline Antivenin vials administered & $1.4 \pm 0.6$ & $1.4 \pm 0.5$ & $1.4 \pm 0.9$ & $0.8802^{\wedge}$ \\
\hline \multicolumn{5}{|l|}{ Outcome, n (\%) } \\
\hline Discharged & 77 (94\%) & 14 (100\%) & $63(93 \%)$ & $0.5821 *$ \\
\hline Expired & $5(6 \%)$ & $0(0 \%)$ & $5(7 \%)$ & \\
\hline Days of care $($ mean $+S D)$ & $1.3 \pm 0.8$ & $1.3 \pm 0.5$ & $1.3 \pm 0.9$ & $0.5488^{\wedge}$ \\
\hline Body temperature $(F)$ & $102.0 \pm 1.4$ & $101.9 \pm 1.3$ & $102.1 \pm 1.5$ & $0.6311^{\#}$ \\
\hline Pulse rate & $133.3 \pm 34.0$ & $151.4 \pm 38.5$ & $129.6 \pm 33.3$ & $0.032 I^{\#}$ \\
\hline Respiration rate & $97.7 \pm 44.4$ & $103.6 \pm 43.5$ & $96.5 \pm 44.8$ & $0.5039^{\wedge}$ \\
\hline SSS & $4.2 \pm 2.5$ & $3.4 \pm 1.3$ & $4.4 \pm 2.7$ & $0.3766^{\wedge}$ \\
\hline \multicolumn{5}{|l|}{ Morbidity rating, n (\%) } \\
\hline Low & $44(54 \%)$ & 10 (71\%) & $34(50 \%)$ & $0.1700 *$ \\
\hline Moderate & $25(30 \%)$ & $4(29 \%)$ & 21 (31\%) & \\
\hline High & $13(16 \%)$ & $0(0 \%)$ & 13 (19\%) & \\
\hline
\end{tabular}

Note: $P$-values were computed by ^Wilcoxon signed-rank test, *Fisher's exact test, or ${ }^{*}$ independent $t$-test. The values are represented as mean \pm standard deviation unless otherwise indicated.

Abbreviation: SSS, snakebite severity score; SD, standard deviation.

and included observation of vital signs, noninvasive blood pressure measurement, and visual inspection for symptoms of immediate hypersensitivity reactions such as development of urticaria, erythema, vomiting, or diarrhea. No adverse reactions were reported with $\mathrm{ACP}$ administration. Additional vials of antivenin were administered to $26 \mathrm{dogs}(24 \%)$. Five dogs $(6 \%)$ died during the course of treatment; no dogs were euthanized. Sixty-five dogs (79\%) were discharged within 24 hours of presentation. Twelve dogs (15\%) were hospitalized for 24-48 hours. Only five dogs $(6 \%)$ were hospitalized for more than 48 hours, including two of the dogs that died.

Forty-four dogs (54\%) received a morbidity score of 1 and were thus classified as having low morbidity. These dogs received a single vial of antivenin and did not develop complications during the course of treatment. Twenty-five dogs $(30 \%)$ received a morbidity score of 2 and were classified as having moderate morbidity. All vaccinated dogs in this study received a morbidity score of 1 or 2 . Thirteen dogs (16\%) received a morbidity score of 3 and were classified as highly morbid; five dogs in this group died. Fresh frozen plasma was used in one case to attempt correction of a coagulation factor deficiency. Packed red blood cells were administered to two dogs to address anemia. One dog died after respiratory arrest during initial stabilization efforts. Two dogs died after respiratory arrest on days 2 and 3 of treatment. One dog had sufficient respiratory compromise to warrant mechanical ventilation and subsequently died of respiratory failure. One dog died acutely of unknown causes on day 3 of treatment. None of the vaccinated dogs in this study died.

There were no statistically significant differences in the clinical presentation between vaccinated and unvaccinated dogs. In unadjusted logistic regression modeling, cases with body weight $\leq 20 \mathrm{~kg}$ (odds ratio $[\mathrm{OR}]=6.5, P=0.0001$ ) or $\mathrm{SSS} \geq 5$ at presentation (OR $=19.1, P<0.0001$ ) had higher morbidity scores. Although not statistically significant, bites to the head were slightly more likely to have higher morbidity scores (OR $=2.5, P=0.0719)$ than bites to the trunk or limbs.

After adjusting for the number of antivenin vials administered, body weight, and bite location, unvaccinated dogs were 2.7 times more likely to have higher morbidity scores, although this estimate was not statistically significant ( $P=0.1673$, Table 3). Vaccination status did not significantly affect the length of hospitalization $(P=0.8119)$ or the number of antivenin vials required for the treatment $(P=0.6923)$. Based on these preliminary results, a power analysis estimates that a total sample size of 400 envenomated dogs, with half having a history of prior vaccination, would be required to prove with statistical vigor that vaccination provides a clinical benefit. 
Table 3 Results of multivariable regression

\begin{tabular}{|c|c|c|c|}
\hline & OR & $(95 \% \mathrm{Cl})$ & $P$-value \\
\hline \multicolumn{4}{|c|}{ Adjusted odds of higher morbidity scores } \\
\hline Unvaccinated status & 2.73 & $(0.66,11.37)$ & 0.1673 \\
\hline Number of antivenin & 5.04 & $(2.15,11.78)$ & 0.0002 \\
\hline \multicolumn{4}{|l|}{ vials administered } \\
\hline Body weight $\leq 20 \mathrm{~kg}$ & 5.89 & $(2.15,16.14)$ & 0.0006 \\
\hline Facial bite location & 1.14 & $(0.37,3.49)$ & 0.8195 \\
\hline
\end{tabular}

Note: Bold values are statistically significant.

Abbreviations: $\mathrm{OR}$, odds ratio; $\mathrm{Cl}$, confidence interval.

\section{Discussion}

Analysis of this population of dogs revealed several factors significantly associated with increased morbidity. Smaller dogs were at significantly increased risk of morbidity; this is consistent with previously published negative correlations between body weight and length of hospitalization. ${ }^{15}$ Bites to the face were slightly more likely to earn a higher morbidity score in this study population, but this did not reach statistical significance. The majority of dogs in this study (74\%) were bitten on the face. This site predilection reflects the naturally inquisitive nature of most dogs, which are likely to approach a rattlesnake and attempt to smell it. Other veterinary studies report a similar proportion of bites to the face. ${ }^{8}$

No statistically significant protective effect of the rattlesnake vaccine was suggested in our data, likely due to the small sample size of vaccinated dogs. The potentially lifesaving benefits of this vaccine merit further study.

This study has several limitations. The study population was limited to moderate to severe cases of envenomation, all of which warranted treatment with antivenin. Dogs that either received a negligible amount of venom during a bite or responded favorably to the vaccine and subsequently developed only mild signs of envenomation would have therefore been excluded. These dogs might not present for emergent care at all, or treatment with antivenin might have been deemed unnecessary by the attending clinician based on presenting clinical signs. This study would also exclude severely envenomated dogs, regardless of vaccination status, for which antivenin therapy was declined for any reason. Similarly, no data were available regarding the specific timing or detailed vaccination history of any dog. Given that the amount of time elapsed since vaccination and the number of vaccines received shape an individual's response to the immunogen in question, an ideal study would use a standardized vaccination protocol and would also be able to control for the time variable between vaccination and envenomation. Finally, the species of rattlesnake responsible for envenomation was not confirmed in any of these cases. In this study, the clinical signs present in all dogs were consistent with envenomation by the western diamondback rattlesnake. The geographic location of this study does overlap with the habitat of the Mojave rattlesnake (Crotalus scutulatus), which the rattlesnake vaccine does not claim to protect against. It is possible that envenomation with a less common species of rattlesnake could confound the current data.

\section{Conclusion}

This preliminary study did not demonstrate a statistically significant protective effect of the canine rattlesnake vaccine in dogs suffering from clinical signs of envenomation warranting treatment with antivenin. Further studies are needed to further explore the appropriate use of this vaccine.

\section{Acknowledgment}

The authors thank Morgan Cavanaugh, Geoff Chapman, Jeanette Yamamoto, and Joan Dolance for help in the preparation of the manuscript.

\section{Disclosure}

The authors report no conflicts of interest in this work.

\section{References}

1. Gold BS, Dart RC, Barish RA. Bites of venomous snakes. $N$ Engl J Med. 2002;347:347-356.

2. JuckettG,Hancox JG. Venomous snakebites in the United States: management review and update. Am Fam Physician. 2002;65:1367-1374.

3. Armentano RA, Schaer M. Overview and controversies in the medical management of pit viper envenomation in the dog. J Vet Emerg Crit Care (San Antonio). 2011;21:461-470.

4. Saviola AJ, Chiszar D, Busch C, Mackessy SP. Molecular basis for prey relocation in viperid snakes. BMC Biol. 2013;11:20.

5. Peterson ME. Snake bite: pit vipers. Clin Tech Small Anim Pract. 2006;21:174-182.

6. Najman L, Seshadri R. Rattlesnake envenomation. Compend Contin Educ Vet. 2007;29:166-176; quiz 176-177.

7. Dart RC, Seifert SA, Carroll L, et al. Affinity-purified, mixed monospecific crotalid antivenom ovine Fab for the treatment of crotalid venom poisoning. Ann Emerg Med. 1997;30:33-39.

8. McCown JL, Cooke KL, Hanel RM, Jones GL, Hill RC. Effect of antivenin dose on outcome from crotalid envenomation: $218 \mathrm{dogs}$ (1988-2006). J Vet Emerg Crit Care (San Antonio). 2009;19:603-610.

9. Antivenin (Crotalidae) Polyvalent (ACP) [product insert], Fort Dodge Laboratories, Inc. Fort Dodge, IA; 2011.

10. C. atrox Toxoid Vaccine Product Information. Available from: http:// www.redrockbiologics.com. Accessed October 14, 2011.

11. Welborn LV, DeVries JG, Ford R, et al. 2011 AAHA canine vaccination guidelines. J Am Anim Hosp Assoc. 2011;47:1-42.

12. Dart RC, Hurlbut KM, Garcia R, Boren J. Validation of a severity score for the assessment of crotalid snakebite. Ann Emerg Med. 1996;27: 321-326.

13. Peterson ME, Matz M, Seibold K, Plunkett S, Johnson S, Fitzgerald K. A randomized multicenter trial of Crotalidae polyvalent immune $\mathrm{F}(\mathrm{ab})$ antivenom for the treatment of rattlesnake envenomation in dogs. J Vet Emerg Crit Care (San Antonio). 2011;21:335-345.

14. Collett D. Modeling Binary Data. 2nd ed. London: Press CHC; 2003.

15. Hackett TB, Wingfield WE, Mazzaferro EM, Benedetti JS. Clinical findings associated with prairie rattlesnake bites in dogs: 100 cases (1989-1998). J Am Vet Med Assoc. 2002;220:1675-1680. 


\section{Publish your work in this journal}

Veterinary Medicine: Research and Reports is an international, Visit http://www.dovepress.com/testimonials.php to read real quotes peer-reviewed, open access journal publishing original research, case reports, editorials, reviews and commentaries on all areas of veterinary medicine. The manuscript management system is completely online and includes a very quick and fair peer-review system.

from published authors.

Submit your manuscript here: http://www.dovepress.com/veterinary-medicine-research-and-reports-journal 